\title{
CONSTITUTIVE MODELLING AND IDENTIFICATION OF PARAMETERS OF 316L STAINLESS STEEL AT CRYOGENIC TEMPERATURES
}

\author{
Maciej RYŚ \\ *Institute of Applied Mechanics, Faculty of Mechanical Engineering, Cracow University of Technology, \\ Al. Jana Pawla II 37, 31-864 Kraków, Poland \\ maciej_rys@02.pl
}

\begin{abstract}
In this work, a macroscopic material model for simulation two distinct dissipative phenomena taking place in FCC metals and alloys at low temperatures: plasticity and phase transformation, is presented. Plastic yielding is the main phenomenon occurring when the yield stress is reached, resulting in nonlinear response of the material during loading. The phase transformation process leads to creation of two-phase continuum, where the parent phase coexists with the inclusions of secondary phase. An identification of the model parameters, based on uniaxial tension test at very low temperature, is also proposed.
\end{abstract}

Key word: Constitutive Modelling; Dissipative Materials; Phase Transformation; Cryogenic Temperature

\section{INTRODUCTION}

The present paper is focused on the constitutive description and identification of the parameters of the model of austenitic stainless steel $316 \mathrm{~L}$ for cryogenic applications. Two coupled dissipative phenomena, plastic flow and phase transformation, are considered using a thermodynamically consistent framework. The theory relies on the notion of local state, and involves one state potential for the state laws, and a dissipation potential for the description of the irreversible phenomena regarded in the model. Parameters of the presented model can be easily identified and the model can be implemented into commercial FEM programs like ABAQUS or ANSYS.

The model presented in this paper describes phase transformation that occurs in metastable stainless steels at very low temperatures. Other phenomena, like discontinuous yielding and damage evolution, are not taken into account here (Egner et al., 2014; Egner, 2013; Egner and Skoczeń, 2010). The FCC (face-centered cubic) to BCC (body-centered cubic) phase transformation has an important meaning in constitutive modelling, because of the influence of the martensitic fraction on hardening process during the plastic deformation. The kinetic laws for state variables are driven from normality rule applied to the plastic potential, while the consistency multiplier is obtained from the consistency condition applied to the yield function (Chaboche, 2008). The classical laws of kinematic and isotropic hardening are postulated in the present work. However, the volume fraction of martensite affects the parameters of both kinematic and isotropic hardening.

The physically based transformation kinetics has been developed by Olson and Cohen (1975). The authors have postulated a three parameter model capable of describing the experimentally verified sigmoidal curve that represents the volume fraction of martensite as a function of plastic strain (Fig. 1):

$\xi=1-\exp \left\{-\beta\left[1-\exp \left(-\alpha \epsilon^{p}\right)\right]^{\lambda}\right.$ where: $\alpha$ denotes the rate of shear-band formation, $\beta$ represents the probability that a shear-band intersection will become a martensite nucleation site, and $\lambda$ is a fixed exponent. The sigmoidal curve is valid for a wide range of temperatures, including room temperature. However, at very low temperatures the rate of phase transformation for an LSFE (low stacking-fault energy) material becomes less temperature dependent, and can be described by a simplified, linearized model (Garion and Skoczeń, 2002):

$\dot{\xi}=A \dot{p} H\left(\left(p-p_{\xi}\right)\left(\xi_{L}-\xi\right)\right)$

In the above equation $A$ is the model parameter, $p$ denotes accumulated plastic strain, $p_{\xi}$ stands for the accumulated plastic strain threshold that triggers the formation of martensite, while $\xi_{L}$ is a limit of martensite content, above which the martensitic transformation rate vanishes. Symbol $H$ denotes the Heaviside step function. Relation (2) introduces a simplified evolution law for the martensite content, with respect to the linear part (region II) of the sigmoidal curve (Fig. 1).

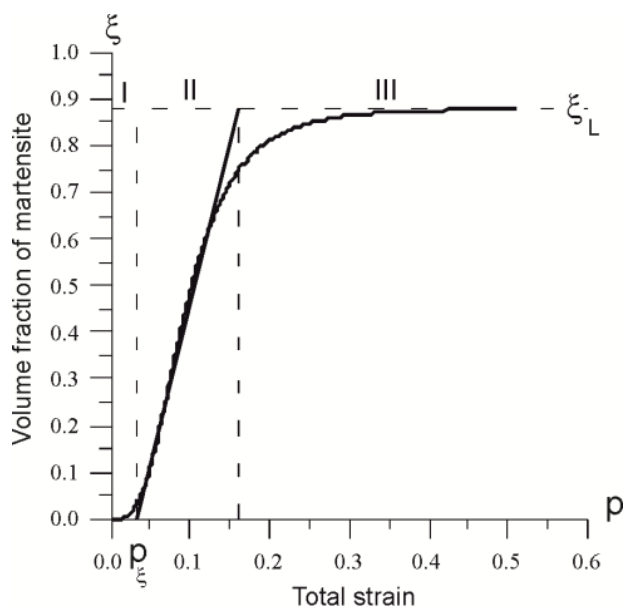

Fig. 1. Volume fraction of $\alpha^{\prime}$ martensite versus accumulated plastic strain (Garion and Skoczeń, 2002) 


\section{CONSTITUTIVE DESCRIPTION OF THE ELASTIC-PLASTIC TWO PHASE MATERIAL}

The author considers a material that is susceptible to two coupled dissipative phenomena: plasticity and phase transformation, that are formalized on the macroscopic level by the use of a proper set of state variables. The motions within the considered thermodynamic system obey the fundamental laws of continuum mechanics (conservation of mass, conservation of linear momentum, conservation of angular momentum) and two laws of thermodynamics, written here in the local form:

- conservation of energy

$\rho \dot{u}-\dot{\epsilon}_{i j} \sigma_{i j}-r+q_{i, i}=0$

- Clausius-Duhem inequality

$\pi=-\rho(\dot{\psi}+s \dot{\theta})+\dot{\epsilon}_{i j} \sigma_{i j}-q_{i} \frac{\theta_{i}}{\theta} \geq 0$

where: $\pi$ denotes the rate of dissipation per unit volume, $\rho$ is the mass density per unit volume; $\sigma_{i j}$ are the components of the stress tensor; $u$ stands for the internal energy per unit mass; $\epsilon_{i j}$ denote the components of the total strain tensor; $r$ is the distributed heat source per unit volume; $q_{i}$ is the outward heat flux; $s$ denotes the internal entropy production per unit mass, $\psi$ stands for Helmholtz' free energy and $\theta$ is the absolute temperature.

The RVE based constitutive model presented in the paper is based on the following assumptions (Egner and Skoczeń, 2010):

1. the martensitic platelets are randomly distributed and randomly oriented in the austenitic matrix;

2. rate independent plasticity is assumed, because the influence of the strain rate is small for the considered range of temperatures (2-77 K) (cf. Hecker et al., 1982);

3. infinitesimal strain theory is applied;

4. mixed isotropic/kinematic plastic hardening affected by the presence of martensite fraction is included;

5. the two-phase material obeys the associated flow rule (volume fraction of new phase not exceeding 0.5 );

6. isothermal conditions are considered (no fluctuations of temperature are taken into account).

Applying infinitesimal deformation theory to elastic - plastic two phase material the total strain $\epsilon_{\mathrm{ij}}$ can be expressed as a sum of the elastic part, $\epsilon_{\mathrm{ij}}^{\mathrm{e}}$ plastic, $\epsilon_{\mathrm{ij}}^{\mathrm{p}}$ and bain strain $\epsilon^{\mathrm{bs}}=1 / 3 \Delta \mathrm{vI}$, denotes the free deformation describing the transformation induced change of the volume, expressed in terms of the relative volume change $\Delta \mathrm{v}$.

$\epsilon_{i j}=\epsilon_{i j}^{e}+\epsilon_{i j}^{p}+\xi \epsilon_{i j}^{b s}$

The presented model is based on the framework of thermodynamics of irreversible processes with internal state variables, where Helmholtz free energy $\psi$ is postulated as a state potential. The state potential depends on the elastic part of the total strain, and set of internal state variables $\mathrm{N}_{k}(k=1,2 \ldots)$, which define the current state of the material:

$\psi=\psi\left(\epsilon_{i j}^{e}, \mathrm{~N}_{k}\right)$

In the case of elastic-plastic material with phase transformation the current state of the material is described by the set of state variables presented in Tab. 1 (cf. Egner, 2012).
Tab. 1. State variables and corresponding thermodynamic forces

\begin{tabular}{|l|c|c|}
\hline \multicolumn{1}{|c|}{ phenomenom } & state variables & conjugated forces \\
\hline $\begin{array}{l}\text { mechanical } \\
\text { variables: total } \\
\text { strain, Cauchy } \\
\text { stress }\end{array}$ & $\frac{\text { observable state }}{\frac{\text { variables }}{\epsilon_{i j}}}$ & $\sigma_{i j}$ \\
\hline $\begin{array}{l}\text { plastic flow } \\
\text { kinematic plastic } \\
\text { hardening } \\
\begin{array}{l}\text { isotropic plastic } \\
\text { hardening } \\
\text { phase } \\
\text { transformation }\end{array}\end{array}$ & $\frac{\text { internal variables }}{\left.\epsilon_{i j}^{e} \text { (or } \epsilon_{i j}^{p}\right)}$ & $\sigma_{i j}$ or $\left(-\sigma_{i j}\right)$ \\
$\alpha_{i j}^{p}$ & $X_{i j}^{p}$ \\
$r^{p}$ & $R^{p}$ \\
\hline
\end{tabular}

The Helmholtz free energy of the material can be written as a sum of elastic $(E)$, inelastic $(I)$ and chemical $(\mathrm{CH})$ terms (Abu Al-Rub and Voyiadjis, 2003; Egner, 2013):

$\psi=\rho \psi^{E}+\rho \psi^{I}+\rho \psi^{C H}$

$\rho \psi^{E}=\frac{1}{2} \epsilon_{i j}^{e} E_{i j k l} \epsilon_{k l}^{e}$

$\rho \psi^{I}=\frac{1}{3} C^{p} \alpha_{i j}^{p} \alpha_{i j}^{p}+R_{\infty}^{p}\left[r^{p}+\frac{1}{b^{p}} \exp \left(-b^{p} r^{p}\right)\right]$

Term $\rho \psi^{C H}$ in Eq. (7) represents the chemically stored energy:

$\rho \psi^{C H}=(1-n) \rho \psi_{\gamma}^{C H}+n \rho \psi_{\alpha \prime}^{C H}$

where: $n$ is a function of martensite content such that $n(0)=0$ and $n(1)=1$ and define general mixture rule. The terms $\rho \psi_{\gamma}^{C H}$ and $\rho \psi_{\alpha \prime}^{C H}$ are the chemical energies of the respective phases, cf. Hallberg et al. (2010), Mahnken and Schneidt (2010). This internally stored energy is different for the two phases and it will affect the generation of heat during phase transformation, as well as the transformation itself.

Using the Clausius-Duhem inequality for isothermal case, one obtains:

$\pi^{\text {mech }}=\sigma_{i j} \dot{\epsilon}_{i j}-\rho \dot{\psi} \geq 0$

where: $\pi^{\text {mech }}$ is defined as mechanical dissipation.

The time derivative of Helmholtz free energy (Eq. 6) as a function of internal state variables is given by:

$\dot{\psi}=\frac{\partial \psi}{\partial \epsilon_{i j}^{e}} \dot{\epsilon}_{i j}^{e}+\frac{\partial \psi}{\partial \alpha_{i j}^{p}} \dot{\alpha}_{i j}^{p}+\frac{\partial \psi}{\partial r^{p}} \dot{r}^{p}+\frac{\partial \psi}{\partial \xi} \dot{\xi}$

Substituting the rate of the Helmholtz free energy into Clausius-Duhem inequality the following thermodynamic constraint is obtained:

$\left(\sigma_{i j}-\rho \frac{\partial \psi}{\partial \epsilon_{i j}^{e}}\right) \dot{\epsilon}_{i j}^{e}+\sigma_{i j} \dot{\epsilon}_{i j}^{p}-\rho \frac{\partial \psi}{\partial \alpha_{i j}^{p}} \dot{\alpha}_{i j}^{p}-\rho \frac{\partial \psi}{\partial r^{p}} \dot{r}^{p}-$

$\rho \frac{\partial \psi}{\partial \xi} \dot{\xi} \geq 0$

Eq. 13 results in the following thermodynamic state laws for the conjugate thermodynamic forces:

$\sigma_{i j}=\rho \frac{\partial \psi}{\partial \epsilon_{i j}^{e}}=E_{i j k l} \epsilon_{k l}^{e}=E_{i j k l}\left(\epsilon_{k l}-\epsilon_{k l}^{p}-\xi \epsilon_{k l}^{b s}\right)$

$X_{i j}^{p}=\rho \frac{\partial \psi}{\partial \alpha_{i j}^{p}}=\frac{2}{3} C^{p} \alpha_{i j}^{p}$ 
$R^{p}=\rho \frac{\partial \psi}{\partial r^{p}}=R_{\infty}^{p}\left[1-\exp \left(-b^{p} r^{p}\right)\right]$

$Z=\rho \frac{\partial \psi}{\partial \xi}=\rho \frac{\partial \psi^{I}}{\partial \xi}+\frac{d n}{d \xi}\left(\rho \psi_{\alpha \prime}^{C H}-\psi_{\gamma}^{C H}\right)$

where: $X_{i j}^{p}, R^{p}$ and $Z$ are the thermodynamic forces conjugated to the state variables $\alpha_{i j}^{p}, r^{p}$ and $\xi$, respectively.

It is assumed here that all dissipative mechanisms are governed by plasticity with a single dissipation potential $F$ (Lemaitre 1992):

$F=F^{p}\left(\sigma_{i j}, X_{i j}^{p}, R^{p}, \xi\right)+F^{t r}(Q, \xi)$

Plastic potential $F^{p}$ is here equal to von Mises type yield surface:

$F^{p}=f^{p}=J_{2}\left(\sigma_{i j}-X_{i j}^{p}\right)-\sigma_{y}-R^{p}$

and the phase transformation dissipation potential is assumed here in a simple form:

$F^{t r}=A Q-B^{t r}=0$

The quantity $Q=\sigma_{i j} \epsilon_{i j}^{b s}-Z$ is conjugated to the transformation rate $\dot{\xi}$ and can be treated as a thermodynamic force that drives the phase front through the material (cf. Hallberg et al., 2007, 2010), $A\left(\theta, \sigma_{i j}, \dot{\epsilon}_{i j}^{p}\right)$, in general, is a function of temperature, stress state and strain rate, and $B^{t r}$ is the barrier force for phase transformation (cf. Mahnken and Schneidt, 2010; Fisher et al., 2000). For rate independent plasticity, isothermal process and small stress variations function $A$ may be treated as a constant value.

Normality rule involves only one plastic multiplier, determined from the consistency condition. The equations involving the dissipation potentials take the form:

$\dot{\epsilon}_{i j}^{p}=\dot{\lambda}^{p} \frac{\partial F^{p}}{\partial \sigma_{i j}}=\dot{\lambda}^{p} \frac{\partial f^{p}\left(\sigma_{k l}, X_{k l}^{p}, R^{p}\right)}{\partial \sigma_{i j}}$

$\dot{\alpha}_{i j}^{p}=-\dot{\lambda}^{p} \frac{\partial F}{\partial \sigma_{i j}}=\dot{\epsilon}_{i j}^{p}$

$\dot{r}^{p=}-\dot{\lambda}^{p} \frac{\partial F^{p}}{\partial R^{p}}=\dot{\lambda}^{p}$

$\dot{\xi}=\dot{\lambda}^{p} \frac{\partial F^{t r}}{\partial Q}=A \dot{p} H\left(\left(p-p_{\xi}\right)\left(\xi_{L}-\xi\right)\right)$

The consistency multiplier $\dot{\lambda}^{p}$ is obtained from the consistency condition:

$\dot{f}^{p}=\frac{\partial f^{p}}{\partial \sigma_{i j}}\left(\dot{\sigma}_{i j}-\dot{X}_{i j}^{p}\right)+\frac{\partial f^{p}}{\partial R^{p}} \dot{R}^{p}+\frac{\partial f^{p}}{\partial \xi} \dot{\xi}=0$

The evolution equations for thermodynamic conjugated forces are obtained by taking time derivatives of quantities defined by equations (14) - (16). In particular, the force rates appearing in consistency condition (25) are given by the following formulae:

$\dot{\sigma}_{i j}=E_{i j k l}\left(\dot{\epsilon}_{k l}-\dot{\epsilon}_{k l}^{p}-\dot{\xi} \epsilon_{k l}^{b s}\right)$

$\dot{X}_{i j}^{p}=\frac{2}{3} C^{p} \dot{\epsilon}_{i j}^{p}+\frac{1}{C^{p}} \frac{\partial C^{p}}{\partial \xi} X_{i j}^{p} \dot{\xi}$

$\dot{R}^{p}=b^{p}\left(R_{\infty}^{p}-R^{p}\right) \dot{r}^{p}+\left[\frac{1}{R_{\infty}^{p}} \frac{\partial R_{\infty}^{p}}{\partial \xi} R^{p}+\left(R_{\infty}^{p}-\right.\right.$ $\left.\left.R^{p}\right) r^{p} \frac{\partial b^{p}}{\partial \xi}\right] \dot{\xi}$

It has to be mentioned here, that in comparison to previous works (Egner and Ryś 2013, Egner et al 2012) the extended evolution equations for thermodynamic conjugate forces (back stress $X_{i j}^{p}$ and drag stress $R^{p}$ ) were derived, accounting for additional terms related to phase transformation rate (full coupling, cf Egner 2013).

\section{IDENTIFICATION OF PARAMETERS OF THE CONSTITUTIVE MODEL}

Identification of the material constants associated with any proposed material model is one of the most challenging issues for researchers, in order to obtain better representation of their material models. If limited test data are available, parameters can be based on the stress-strain data obtained from the uniaxial tension or compression experiments. In the present work a uniaxial tension test for $316 \mathrm{~L}$ stainless steel at temperature $4.2 \mathrm{~K}$ is used to determine functions $C^{p}(\xi), b^{p}(\xi)$ and $R_{\infty}^{p}(\xi)$ occurring in the kinetic laws (Eq. (27) $-(28))$. The simplest, linear form of these functions is here assumed, namely:

$C^{p}(\xi)=C_{0}^{p}\left(1+h_{C} \xi\right)$

$R_{\infty}^{p}(\xi)=R_{\infty, 0}^{p}\left(1+h_{R} \xi\right)$

$b^{p}(\xi)=b_{0}^{p}\left(1+h_{b} \xi\right)$

Eventually the following parameters have to be determined in the present model: $C_{0}^{p}, h_{C}, R_{\infty, 0}^{p}, h_{R}, b_{0}^{p}, h_{b}$. The value of the parameter $A \quad(24)$ is taken from the paper by Garion et al. (2006). The function which describes the experimental results of martensite content was found in the following form (Garion et al, 2006), (Fig. 2):

$\xi=4.3714 p-0.3873$, for $p>p_{\xi}$

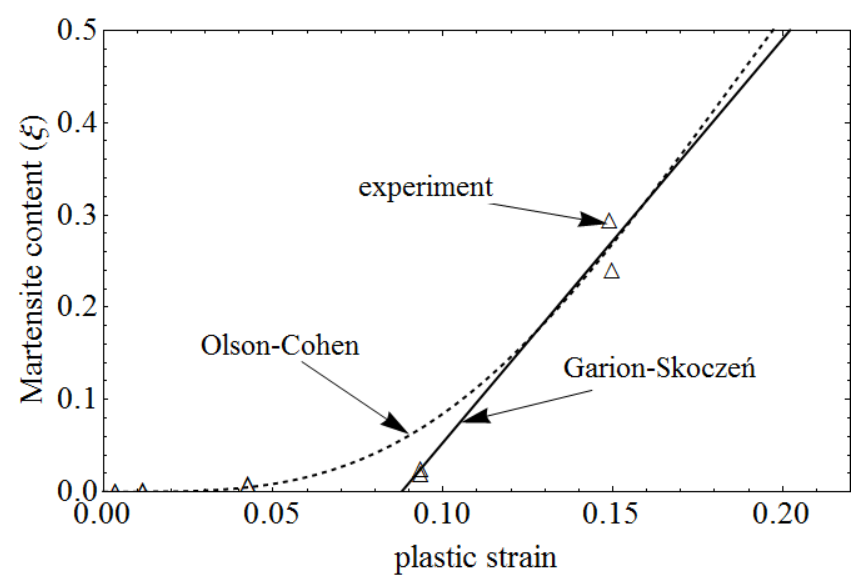

Fig. 2. Martensite content as a function of plastic strain

As was mentioned before, determining of all parameters occurring in kinetic equations is based on the experimental stressstrain curve obtained for $316 \mathrm{~L}$ stainless steel at $4.2 \mathrm{~K}$. The test was performed with use of the cryostat filled up with liquid helium and equipped with tensometers, extensometers and a load cell aligned with the sample. It was concerned that the kinematically controlled tensile test is the most suitable. Moreover, the measurement of the volume fraction of martensite was based on ferromagnetic properties of the BCC martensitic phase whereas the FCC austenitic matrix is paramagnetic. It has to be mentioned 
here that the experiments carried out in liquid helium are expensive and laborious and this is the reason why the identification of the parameters is based only on uniaxial tension test. The stress strain curve was divided into three regions: elastic, plastic and plastic with phase transformation. Every point on the curve within plastic region can be described in the following way (Fig. 3. range II, see also Abu Al-Rub 2004, Lemaitre 1992):

$$
\sigma=\sigma_{y}+X^{p}\left(\epsilon^{p}\right)+R^{p}(p)
$$

where: $\sigma_{y}$ is the yield stress and equations for kinematic $X^{p}\left(\epsilon^{p}\right)$, and isotropic, $R^{p}(p)$, hardening in the case of uniaxial state of stress are exspressed as:

$X=C_{0}^{p} \epsilon^{p} ; R^{p}=b_{0}^{p}\left(R_{\infty, 0}^{p}-R^{p}\right) p$

Now the standard least-square minimization method was used to find the parameters $C_{0}^{p}, R_{\infty, 0}^{p}, b_{0}^{p}$ and fit the model to the data points.

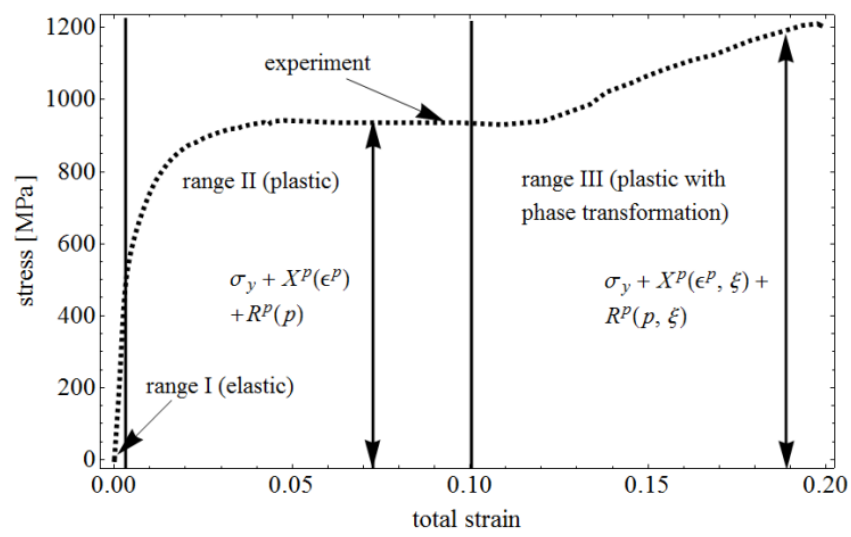

Fig. 3. Stress-strain curve for $316 \mathrm{~L}$ stainless steel at $4.2 \mathrm{~K}$

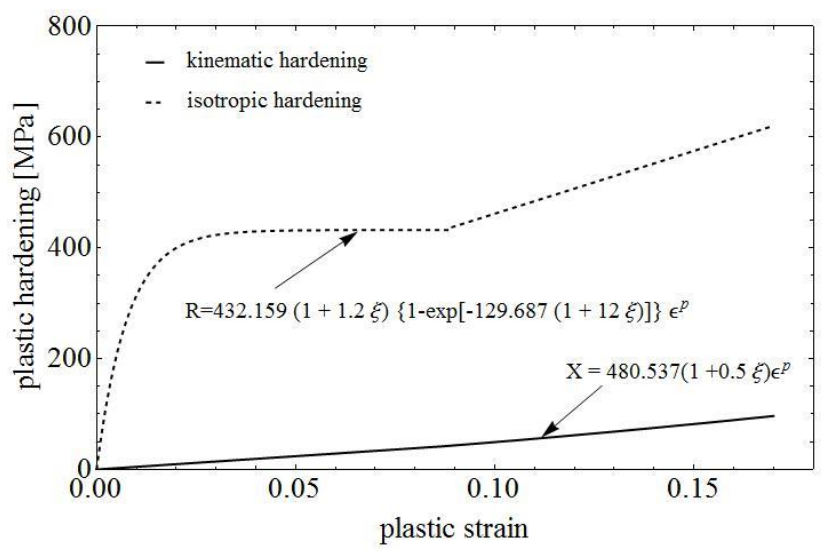

Fig. 4. Evolution of the plastic hardening forces

Every point within inelastic region can be described as follows (Fig. 3. Range II and III):

$\sigma=\sigma_{y}+X^{p}\left(\xi, \epsilon^{p}\right)+R^{p}(\xi, p)$

where:

$X^{p}\left(\xi, \epsilon^{p}\right)=C_{0}^{p}\left(1+h_{C} \xi\right) \epsilon^{p}$

$R^{p}(\xi, p)=b_{0}^{p}\left(1+h_{b} \xi\right)\left(R_{\infty, 0}^{p}\left(1+h_{R} \xi\right)-R^{p}\right) p$ where: $p$ is equal to $\epsilon^{p}$ in the case of uniaxial tension and parameters: $C_{0}^{p}, R_{\infty, 0}^{p}, b_{0}^{p}$ are already known. Again, the standard least-square minimization method was used to find the rest of the unknown parameters. All values of the identified parameters are listed in Tab. 2. The curves plotted in Fig. 4 represent the synergetic effects of the combined isotropic and kinematic hardening associated with plasticity found for the present model.

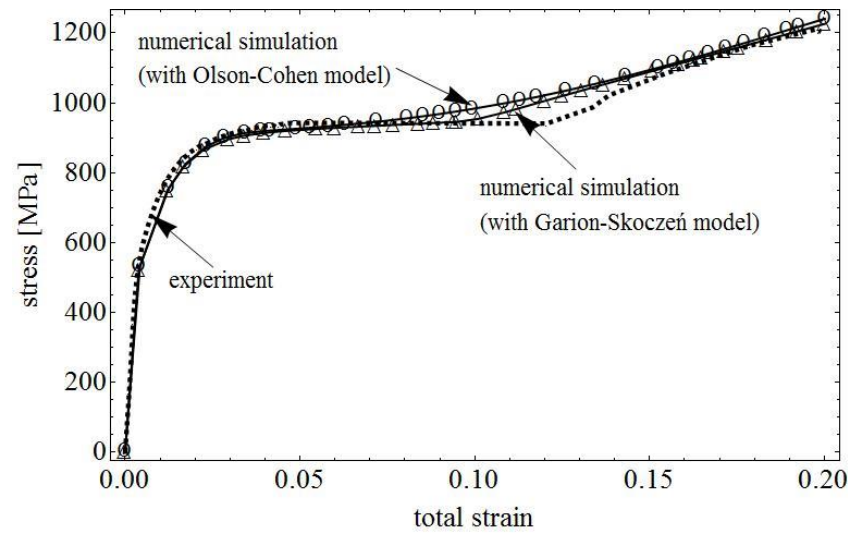

Fig. 5. Stress-strain curve for 316L stainless steel

Accounting for two dissipative phenomena: plasticity and phase transformation in the present constitutive model allows to obtain a satisfactory reproduction of the experimental stressstrain curve for $316 \mathrm{~L}$ stainless steel subjected to uniaxial tension at cryogenic temperatures (see Fig. 5). A small difference between numerical and experimental results is caused by damage which is not included in the present model. Using Garion-Skoczen linear kinetic law of phase transformation also exacerbates the numerical results. However, the model presented here is easy to identify experimentally because the number of material parameters is reasonably small. It should be pointed out that the experiments carried out in liquid helium or liquid nitrogen are laborious, expensive and usually require complex cryogenic installations to maintain stable conditions (constant or variable temperature). Therefore, any justified simplification leading to reduction of the number of parameters to be determined is of great importance.

Tab. 2 Material data for $316 \mathrm{~L}$ stainless steel at the temperature of $4.2 \mathrm{~K}$

\begin{tabular}{|l|r|}
\hline Young modulus [GPa] & 176.818 \\
Poisson ratio & 0.3 \\
Proportionality limit [MPa] & 470 \\
$C_{0}^{p}[\mathrm{MPa}]$ & 480.537 \\
$R_{\infty, 0}^{p}[\mathrm{MPa}]$ & 432.159 \\
$b_{0}^{p}$ & 129.687 \\
$h_{C}$ & 0.5 \\
$h_{R}$ & 1.2 \\
$h_{b}$ & 12 \\
$\Delta v$ & 0.02 \\
$p_{\xi}$ & 0.0886 \\
$\xi_{L}$ & 0.9 \\
$A$ & 4.3714 \\
\hline
\end{tabular}




\section{CONCLUSIONS}

The constitutive model presented in the paper includes two dissipative phenomena: plastic yielding and plastic strain induced phase transformation. A consistent thermodynamic framework was used in order to describe dissipative phenomena. Two kinetic laws of phase transformation were used: Olson-Cohen and Garion-Skoczeń model. As shown in Fig. 5 and Fig. 2, the use of simplified Garion-Skoczeń linear phase transformation law allows for a very good approximation of the second stage of OlsonCohen sigmoidal curve (see Fig. 1) and a stress-strain curve. A great advantage of the presented model is a relatively small amount of parameters that can be determined in the simple way. A standard uniaxial tension test and least squares method were used to identify the model parameters. Validation of the model is based on the available experimental data and very rare experiments carried out at extremely low temperatures. Such tests are extremely laborious, complex, costly and time consuming and are not common even in the centers, where the low temperature research belongs to the standard activities (like CERN). For this reason, given the scarce experimental background, the model has been validated on one single test only. As the results, however, seem to be very encouraging, the author believes that the model performs correctly and can be further applied to a larger class of problems of low temperature material behaviour. As soon as some more experimental data is available, the validation of the model will certainly be confirmed.

\section{REFERENCES}

1. Abu Al Rub R. K (2004), Material length scales in gradientdependent plasticity/damage and size effects: theory and computation, Ph.D. Thesis, , Lousiana State University, Louisiana, USA.

2. Abu Al Rub R. K, Voyiadjis G.Z. (2003), On the coupling of anisotropic damage and plasticity models for ductile materials, International Journal of Plasticity, 40, 2611-2643.

3. Chaboche, J. (2008), A review of some plasticity and viscoplasticity constitutive theories, International Journal of Plasticity, 24, 1642-1693.

4. Egner H. (2012), On the full coupling between thermo-plasticity and thermo-damage in thermodynamic modeling of dissipative materials, International Journal of Solids and Structures, 34, 61-92.

5. Egner H. (2013), Constitutive modelling of coupled problems of dissipative materials mechanics, Seria Mechanika, Monografia 444, Kraków (in Polish).
6. Egner H., Egner W., Ryś M. (2012), Thermodynamics-based constitutive modeling of coupled dissipative phenomena in engineering materials, Research and Applications in Structural Engineering, Mechanics and Computation - Zingoni, Taylor\&Francis Group, London

7. Egner H., Ryś M. (2012), Modeling of coupling between damage and phase transformation in austenitic stainless steel at cryogenic temperatures, Czasopismo Techniczne, 8-M/2012, 22, (in Polish).

8. Egner H., Skoczeń B. (2010), Ductile damage development in twophase materials applied at cryogenic temperatures, International Journal of Plasticity, 26, 488-506.

9. Egner H., Skoczeń B., Ryś M. (2014), Constitutive and numerical modeling of coupled dissipative phenomena in $316 \mathrm{~L}$ stainless steel at cryogenic temperatures, International Journal of Plasticity, 64, 113-133.

10. Fischer, F.D., Reisner, G., Werner, E., Tanaka, K., Cailletaud, G., Antretter, T. (2000), A new view on transformation induced plasticity (TRIP), International Journal of Plasticity, 16(1-8), 723-748.

11. Garion C., Skoczeń B. (2002), Modeling of plastic strain induced martensitic transformation for cryogenic applications, Journal of Applied Mechanics, 69, 6, 755-762.

12. Garion C., Skoczeń B., Sgobba S. (2006), Constitutive modelling and identification of parameters of the plastic strain induced martensitic transformation in $316 \mathrm{~L}$ stainless steel at cryogenic temperatures, International Journal of Plasticity, 22, 7, 1234-1264.

13. Hallberg H., Hakansson P., Ristinmaa M. (2010), Thermomechanically coupled model of diffusionless phase transformation in austenitic steel, International Journal of Solids and Structures, 47, 1580-1591.

14. Hallberg, H., Hakansson, P., Ristinmaa M. (2007), A constitutive model for the formation of martensite in austenitic steels under large strain plasticity, International Journal of Plasticity, 23, 1213-1239.

15. Hecker S. S., Stout M. G., Staudhammer K. P., Smith J. L. (1982), Effects of strain state and strain rate on deformation-induced transformation in 304 stainless steel: Part I. Magnetic measurements and mechanical behaviour. Metallurgical Transactions, A 13A, 619626.

16. Lemaitre H. (1992), A course on damage mechanics. SpringerVerlag, Berlin and New York.

17. Mahnken, R., Schneidt, A. (2010), A thermodynamics framework and numerical aspects for transformation-induced plasticity at large strains, Archives of Applied Mechanics, 80, 229-253.

18. Olson, G. B., Cohen, M. (1975), Kinetics of strain-induced martensitic nucleation, Metallurgical Transactions, 6A, 791-795.

Acknowledgments: This work has been supported by the National Science Centre through the Grant No. 2013/11/B/ST8/00332. 